\title{
Splenic volume as a prognostic indicator for unresectable pancreatic cancer
}

\author{
REI SUZUKI ${ }^{1}$, SHIRO ISHII $^{2}$, HIROTAKE WATANABE $^{2}$, TADAYUKI TAKAGI $^{1}$, \\ MITSURU SUGIMOTO ${ }^{1}$, YUKI SATO ${ }^{1}$, JUN NAKAMURA ${ }^{3}$, MIKA TAKASUMI ${ }^{1}$, TSUNETAKA KATO ${ }^{1}$, \\ MINAMI HASHIMOTO ${ }^{3}$, TAKUTO HIKICHI $^{3}$, HIROSHI ITO $^{2}$ and HIROMASA OHIRA ${ }^{1}$ \\ Departments of ${ }^{1}$ Gastroenterology, ${ }^{2}$ Radiology and ${ }^{3}$ Endoscopy, \\ Fukushima Medical University School of Medicine, Fukushima 960-1295, Japan
}

Received November 12, 2020; Accepted December 16, 2020

DOI: $10.3892 /$ wasj.2020.80

\begin{abstract}
The clinical role of an enlarged spleen in pancreatic cancer (PC) patients treated with chemotherapy has not yet been fully evaluated. Thus, the present study investigated the association between splenic volume and prognosis in 50 patients with unresectable PC treated with chemotherapy. Contrast-enhanced computed tomography was performed prior to chemotherapy in all patients. A 3D reconstruction of the spleen and splenic volume measurements was performed by an experienced radiologist. Patients were divided into 2 groups based on reported splenic volume and the cut-off value set at the mean +1 standard deviation (SD) as follows: Group $1(\mathrm{n}=34$, splenic size $<$ mean $+1 \mathrm{SD})$ and group 2 $(n=16$, splenic size $\geq$ mean $+1 S D)$. Survival analysis for overall survival (OS) was performed using the Kaplan-Meier method with the log-rank test in univariate analysis. Forward stepwise multivariate analysis was performed to determine the influence of clinicopathological variables. Cox regression analysis was used for multivariate analysis, and hazard ratios (HRs) were calculated. A P-value $<0.05$ was considered to indicate a statistically significant difference. In the survival analysis, splenic volume (group 1 vs. group 2, median of 14.8 vs. 8.2 months, $\mathrm{P}=0.04$ ) was related to $\mathrm{OS}$. The results of multivariate Cox regression analysis confirmed that treatment $(\mathrm{P}=0003), \mathrm{N}$-stage $(\mathrm{P}=0.001), \mathrm{M}$-stage $(\mathrm{P}=0.0001)$ and splenic volume $(\mathrm{P}=0.001)$ were independent prognostic factors for OS. A higher incidence of severe thrombocytopaenia was observed in group 2 than in group 1 . On the whole, the present study demonstrates that splenic volume may be a prognostic indicator for patients with PC.
\end{abstract}

Correspondence to: Dr Rei Suzuki, Department of Gastroenterology, Fukushima Medical University School of Medicine, 1 Hikarigaoka, Fukushima 960-1295, Japan

E-mail: subaru@fmu.ac.jp

Key words: pancreatic cancer, chemotherapy, splenic volume, prognosis, thrombocytopenia

\section{Introduction}

Left-sided portal hypertension is a rare clinical syndrome. While left-sided portal hypertension can be caused by every disease entity involving extrahepatic portal vein system obstruction or stricture, pancreatic diseases, such as pancreatitis and pancreatic neoplasm are reported to be major etiologies (1-4). Left-sided portal hypertension can induce various clinical manifestations, including abdominal pain and gastrointestinal bleeding. An enlarged spleen is often observed in patients with portal hypertension, although the incidence of an enlarged spleen in left-sided portal hypertension has not yet been described, at least to the best of our knowledge.

In solid tumors, an enlarged spleen or splenomegaly is often observed following the administration of certain types of chemotherapeutic agents $(5,6)$. Among these agents, oxaliplatin (L-OHP) is a well-known inducer of splenic enlargement due to sinusoidal injury. Moreover, it has been reported that the splenic volume prior to L-OHP treatment is related to thrombocytopenia, as well as to prognosis. The increased incidence of thrombocytopenia may be attributed to the splenic sequestration of platelets, as well as to the direct suppression of the bone marrow by the drug (6). More recently, Aarnink et al evaluated the prognostic role of splenic volume in patients with pancreatic cancer (PC) treated with an L-OHP-containing regimen (FOLFIRINOX) [combination of L-OHP, irinotecan (CPT-11) and fluorouracil (5-FU)] (7). In their study, they found that a large pre-treatment splenic volume was an independent prognostic indicator, together with other indicators, such as performance status, liver metastasis and baseline tumour markers; however, the mechanisms underlying the poor prognosis of patients with a large splenic volume have not been clearly described. Moreover, the clinical role of an enlarged spleen in patients with PC treated with other chemotherapeutic regimens has not yet been evaluated, at least to the best of our knowledge.

To determine this involvement, the present study investigated the association between splenic volume and prognosis in patients with PC treated with various chemotherapeutic regimens. 


\section{Patients and methods}

Study design and patient treatment. A retrospective cohort study was conducted, reviewing data from patients diagnosed with PC, including locally advanced and metastatic disease, at Fukushima Medical University between April, 2014 and December, 2019. Patients with histopathologically confirmed $\mathrm{PC}$ with sufficient imaging data for $3 \mathrm{D}$ reconstruction and splenic volume measurement were included, whereas those who were assumed to have PC based on imaging findings or serum tumour marker levels were excluded from the study. Patients who had a known diagnosis of chronic liver disease (hepatitis, liver cirrhosis and hepatocellular carcinoma) were also excluded. Additionally, patients with rare primary pancreatic neoplasms, including acinar cell carcinoma or neuroendocrine carcinoma, were excluded. Patients who underwent conversion surgery were excluded. All patients were chemotherapy-naïve and standard treatment with gemcitabine (GEM), S-1, gemcitabine plus S-1, gemcitabine plus radiation therapy, gemcitabine plus nab-paclitaxel $(\mathrm{GnP})$ therapy or FOLFIRINOX (FFX) was initiated. Treatment with gemcitabine or S-1 alone was defined as monotherapy, and the other regimens were defined as combination therapy.

Adverse events were graded according to the Common Terminology Criteria for Adverse Events version 5.0 (CTCAE ver.5) (8). Severe hematotoxicity was defined as adverse events of a grade $>3$ according to the CTCEA ver.5. Treatment discontinuation was defined as the discontinuation of treatment due to severe hematotoxicity at least once during the treatment.

Splenic volume was first evaluated in 63 patients who met the inclusion criteria mentioned above. Among the 63 patients, 13 patients were excluded from the evaluation due to the following reasons: Early termination of the 1st cycle of chemotherapy in 6 patients, post-operative status in 5 patients, concomitant existence of another cancer in 1 patient and data duplication in 1 patient (Fig. 1). Ultimately, 50 patients were included in the present study. The clinical background of the patients is summarized in Table I. Briefly, the median age was 66.0 years (range, 42.0-85.0 years), the median height was $150.5 \mathrm{~cm}$ (range, 138.6-178.0 cm) and the median body weight was $48.7 \mathrm{~kg}$ (range, 34.4-78.0 kg). The patients included 22 males and 28 females. A total of 23 were diagnosed with clinical stage III disease $(46.0 \%)$. The treatments included 1st-line chemotherapy with gemcitabine monotherapy in 16 patients, S-1 monotherapy in 4 patients, $\mathrm{GnP}$ in 20 patients, FFX in 8 patients, gemcitabine plus radiation therapy in 1 patient and gemcitabine plus S-1 in 1 patient.

All clinicopathological data, including age, sex, height, body weight, location of the disease, clinical stage, serum levels of carcinoembryonic antigen (CEA) and carbohydrate antigen 19-9 (CA19-9), white blood cell counts, red blood cell counts, platelet counts, the neutrophil-to-lymphocyte ratio (NLR), and serum levels of aspartate aminotransferase (AST) and alanine aminotransferase (ALT), were measured immediately prior to the initial chemotherapy. Clinical stage was determined according to the American Joint Committee on Cancer (AJCC)/Union for International Cancer (UICC) staging system, version 8 (9). The percentage of planned drug intensity delivered for each drug was also calculated and reported as the relative drug intensity (RDI). In a previous
Table I. Clinical characteristics of the included patients.

\begin{tabular}{lc}
\hline Variables & $\begin{array}{c}\text { Number of patients } \\
(\mathrm{n}=50)\end{array}$ \\
\hline Age, years & $66.0(42.0-85.0)$ \\
Height $(\mathrm{cm})$ & $159.5(138.6-178.0)$ \\
Body weight $(\mathrm{kg})$ & $48.7(34.4-78.0)$ \\
Sex, male, $\mathrm{n}(\%)$ & $22(44.0)$ \\
Location of disease, Ph, n (\%) & $33(66.0)$ \\
cStage, I-III, n (\%) & $23(46.0)$ \\
Treatment & \\
GEM & 16 \\
S-1 & 4 \\
GnP & 20 \\
FFX & 1 \\
GEM + RT & 1 \\
GS & $138.2(39.7-343.7)$ \\
Splenic volume (cm $\left.{ }^{3}\right)$ & \\
\hline Ph, head of the pancreas; GEM, gemcitabine; GnP, gemcitabine \\
plus nab-paclitaxel; FFX, FOLFIRINOX; RT, radiotherapy; GS, \\
gemcitabine plus S-1.
\end{tabular}

study, splenic volume measured by $\mathrm{CT}$ scan was reported to be $127.4+62.9 \mathrm{~cm}^{3}$ (mean +1 standard deviation) in the Japanese population (10). Therefore, this value was utilized to divide the patients into 2 groups as follows: Group 1 (splenic size $<127.4+62.9 \mathrm{~cm}^{3}$ ) and group 2 (splenic size $\left.\geq 127.4+62.9 \mathrm{~cm}^{3}\right)$.

The study protocol conformed to the ethical guidelines of the 1975 Declaration of Helsinki and was approved by the Institutional Review Board of Fukushima Medical University (IRB \#29254). The institutional review board waived the need for written informed patient consent due to the retrospective and non-interventional nature of the study.

CT imaging protocols. Abdominal CT examinations were performed with or without contrast enhancement, with singleor triple-phase scanning. The acquisition parameters were as follows: 64- and 320-channel multidetector row scanners (Aquilion 64 and Aquilion one, Toshiba Medical Systems), helical scan mode, tube voltage of $135 \mathrm{kVp}$, variable tube current (in $\mathrm{mA}$; autoexposure), $0.5 \mathrm{sec} /$ rotation, $0.5 \mathrm{~mm}$ collimation, and a pitch of 41 for Aquilion 64 and 51 for Aquilion one. Images were reconstructed at a contiguous axial $1 \mathrm{~mm}$ thickness. Contrast material was administered by an intravenous injection of $100 \mathrm{ml}$ using a power injector (Nemotokyorindo) at a rate of $3.3 \mathrm{ml} / \mathrm{sec}$ with acquisition delays of $30 \mathrm{sec}, 45 \mathrm{sec}$ and $120 \mathrm{sec}$ in dynamic $\mathrm{CT}$ or a rate of $1 \mathrm{ml} / \mathrm{sec}$ with a 150 -sec delay in single-phase CT.

$3 D$ reconstruction and splenic volume measurement. $\mathrm{CT}$ images obtained at the authors' institution and before chemotherapy was commenced were included in the evaluation, and the splenic volume was measured in 63 patients. The volume of the spleen was measured with the volume rendering technique from a 


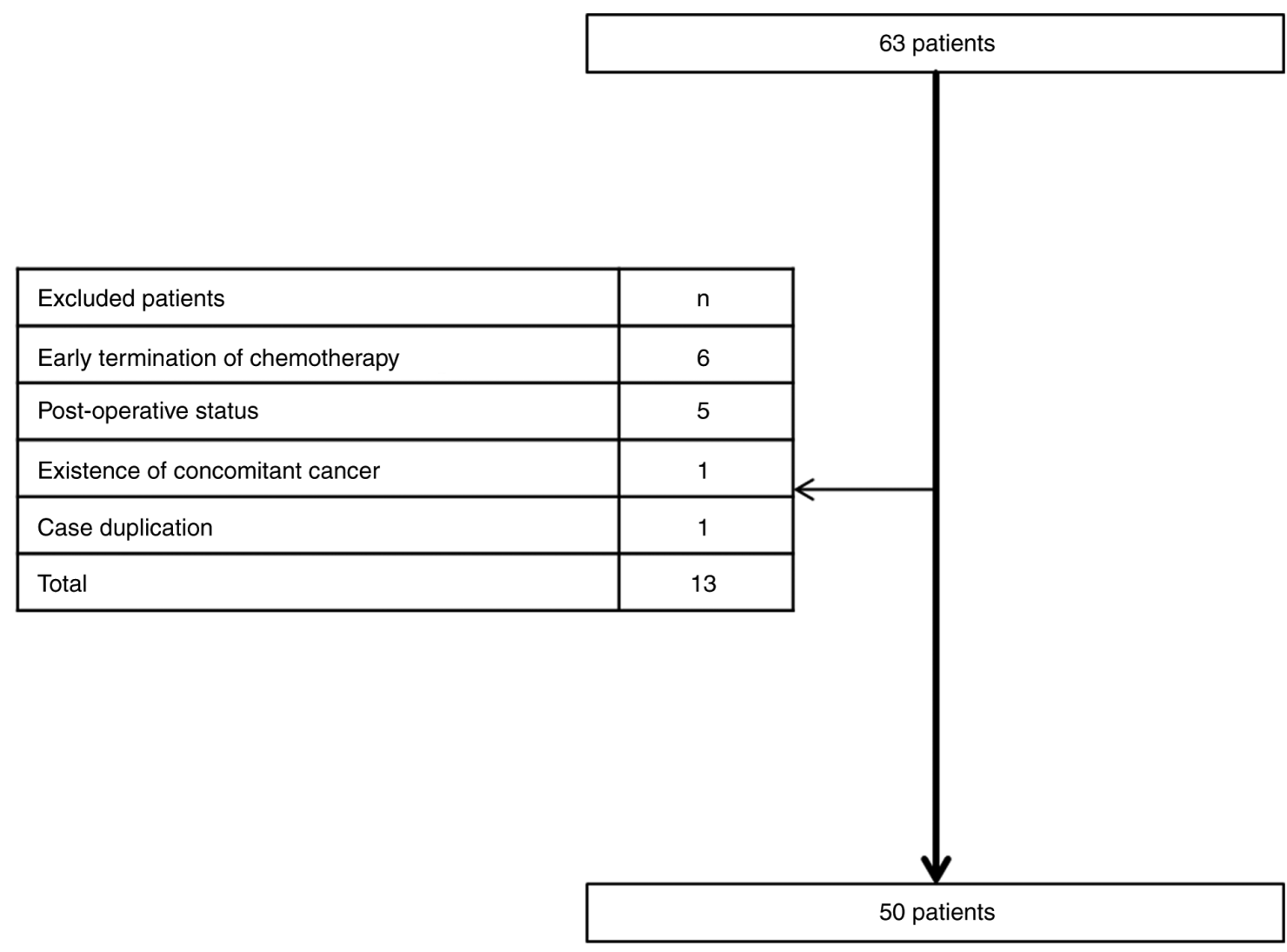

Figure 1. Flow diagram schematic illustrating patient enrolment.

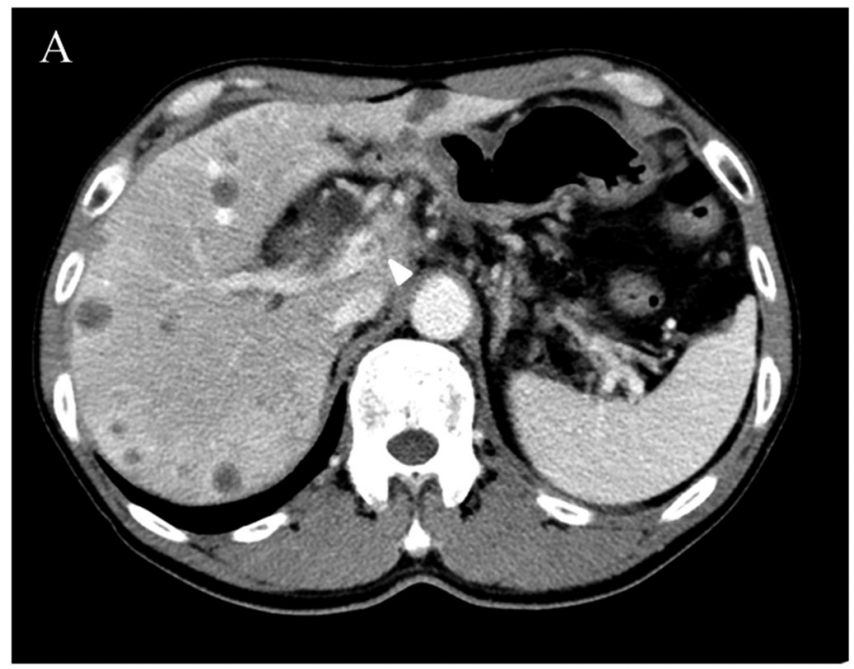

B

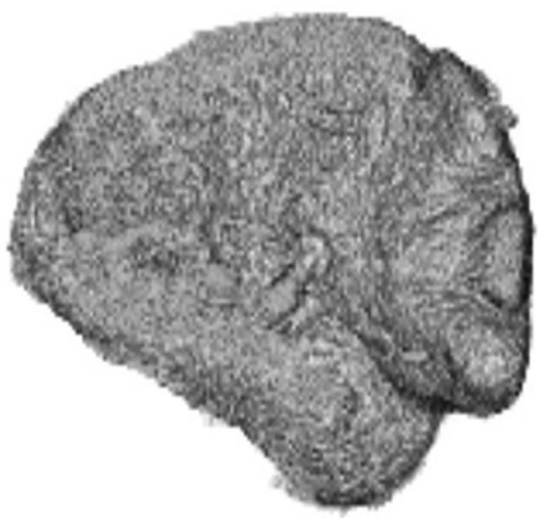

Splenic volume $207.5 \mathrm{~cm}^{3}$

Figure 2. Image of 3D reconstruction of the spleen. (A) CT image of patients with portal vein invasion and enlarged spleen. Arrowhead indicates portal vein. (B) Images of 3D reconstruction of the enlarged spleen. Splenic volume was $207.5 \mathrm{~cm}^{3}$. 3D, 3-dimensional.

1-mm slice of CT data using ziostation2 (Ziosoft Inc.). When the correct image of the spleen was selected, the volume of the spleen was calculated automatically (Fig. 2).

Assessment of vascular invasion. Invasion of the portal vein, supra mesenteric vein and splenic vein was evaluated by board-certified radiologists with 19 years of experience in CT imaging. CT images were interpreted using a multiplanar reformation tool that allows the assessment of axial and coronal source images. The reader of the CT scan was also blinded to all other information except the purpose of the present study. To quantify the extent of vascular invasion, 0 points were scored for no vascular involvement, 1 point for mild stricture of vessels or contact without stricture, 2 points for severe stricture and 3 points for vascular obstruction at the portal vein, supra mesenteric vein and splenic vein, and the scores were added to obtain the portal vein system invasion score (invasion score, range, 0-9 points). 
Table II. Comparison of clinical characteristics between patients in groups 1 and 2.

\begin{tabular}{|c|c|c|c|}
\hline Variables & Group $1(n=34)$ & Group $2(n=16)$ & P-value \\
\hline Age, years & $66.5(42.0-85.0)$ & $63.5(45.0-79.0)$ & 0.15 \\
\hline Height (cm) & $159.5(138.6-178.0)$ & $160.3(146.0-177.6)$ & 0.98 \\
\hline Body weight (kg) & $46.9(34.4-72.4)$ & $54.5(36.5-78.0)$ & 0.32 \\
\hline Body surface area $\left(\mathrm{m}^{2}\right)$ & $1.5(1.2-1.8)$ & $1.6(1.3-1.9)$ & 0.35 \\
\hline Sex, male, $\mathrm{n}(\%)$ & $18(52.9)$ & $10(62.5)$ & 0.55 \\
\hline Location of disease, $\mathrm{Ph}, \mathrm{n}(\%)$ & $24(70.5)$ & $9(56.5)$ & 0.32 \\
\hline cStage, I-III, n (\%) & $18(52.9)$ & $7(43.7)$ & 0.76 \\
\hline Treatment, monotherapy (\%) & $13(38.2)$ & $9(56.2)$ & 0.36 \\
\hline T-stage, T1-3 (\%) & $11(32.3)$ & $5(31.2)$ & .99 \\
\hline N-stage, N0 (\%) & $6(17.6)$ & $1(6.2)$ & 0.40 \\
\hline M-stage, M0 (\%) & $13(38.2)$ & $9(56.2)$ & 0.36 \\
\hline Portal vein invasion, $\mathrm{n}(\%)$ & $9(26.4)$ & $10(62.5)$ & 0.01 \\
\hline Supra mesenteric vein invasion, $\mathrm{n}(\%)$ & $11(32.3)$ & $12(75.0)$ & 0.005 \\
\hline Splenic vein invasion, $\mathrm{n}(\%)$ & $10(29.4)$ & $10(62.5)$ & 0.02 \\
\hline $\mathrm{CEA}(\mathrm{ng} / \mathrm{ml})$ & $4.3(1.4-80.3)$ & $3.1(1.2-1,738.0)$ & 0.05 \\
\hline CA19-9 (U/ml) & $1,390.0(0.3-69,527)$ & $185.3(0.9-60,300)$ & 0.07 \\
\hline WBCs $\left(/ \mathrm{mm}^{3}\right)$ & $6,050.0(3,500-14,900)$ & $5,100.0(3,100.0-10,500.0)$ & 0.18 \\
\hline Neutrophils $\left(/ \mathrm{mm}^{3}\right)$ & $4,230.0(2,052.0-12,814.0)$ & $3,417.0(1,395.0-8,610.0)$ & 0.28 \\
\hline Haemoglobin & $12.5(8.1-15.1)$ & $13.0(8.9-14.1)$ & 0.19 \\
\hline Platelets $\left(\mathrm{x} 10^{4} / \mathrm{mm}^{3}\right)$ & $20.4(8.6-48.0)$ & $18.8(6.5-54.2)$ & 0.40 \\
\hline NLR & $3.6(1.4-9.6)$ & $3.7(1.0-9.3)$ & 0.88 \\
\hline $\operatorname{AST}(\mathrm{U} / \mathrm{ml})$ & $20.0(10.0-106.0)$ & $21.0(16.0-220.0)$ & 0.44 \\
\hline $\operatorname{ALT}(\mathrm{U} / \mathrm{ml})$ & $20.5(7.0-238.0)$ & $25.0(8.0-213.0)$ & 0.50 \\
\hline Splenic volume $\left(\mathrm{cm}^{3}\right)$ & $102.2(39.7-175.3)$ & $227.9(190.1-343.7)$ & $<0.001$ \\
\hline
\end{tabular}

CEA, carcinoembryonic antigen; CA19-9, carbohydrate antigen 19-9; AST, aspartate aminotransferase; ALT, alanine aminotransferase. Mann-Whitney test was used for the analysis of for continuous variables and Fisher's exact test for categorical variables.
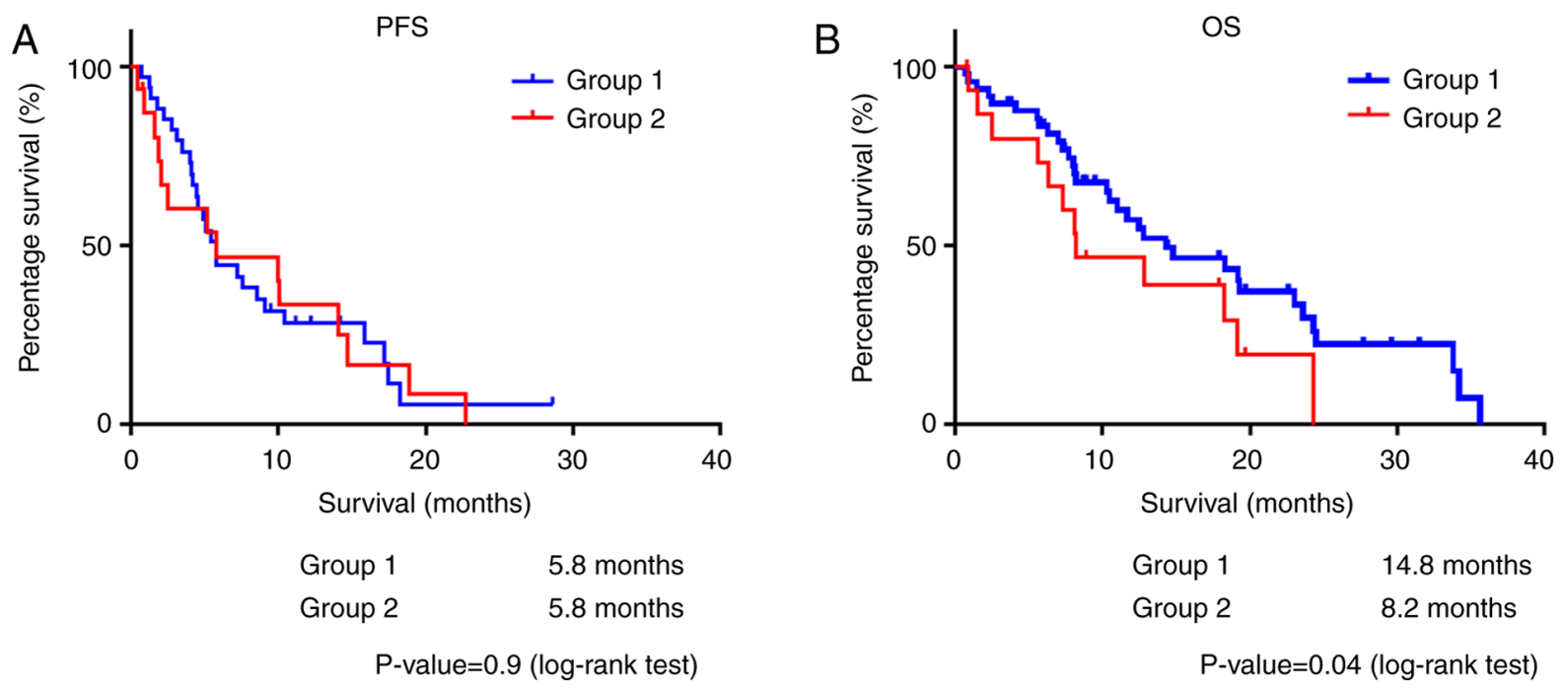

Figure 3. Survival analysis between Group 1 and Group 2. (A) As for PFS, there was no significant difference between the 2 groups (P=0.9). (B) The OS of patients in group 2 was lower than that of patients in group $1(14.8 \mathrm{vs} .8 .2$ months, $\mathrm{P}=0.04)$. Group 1 (splenic size $\left.<127.4+62.9 \mathrm{~cm}^{3}\right)$ and group 2 (splenic size $\left.\geq 127.4+62.9 \mathrm{~cm}^{3}\right)$. PFS, progression-free survival; OS, overall survival.

Statistical analyses. Continuous variables (i.e., age, height, body weight, body surface area, CEA, CA19-9, WBC and platelets counts, hemoglobin, NLR, AST, ALT and splenic volume) are reported as the median and range and were compared using a Mann-Whitney test. Categorical variables (i.e., sex, location of disease, cStage, treatment and portal vein 
Table III. Results of survival analysis.

\begin{tabular}{|c|c|c|c|c|c|c|c|c|}
\hline \multirow[b]{3}{*}{ Variables } & \multicolumn{4}{|c|}{ PFS } & \multicolumn{4}{|c|}{ OS } \\
\hline & \multirow[b]{2}{*}{ Median } & \multicolumn{2}{|c|}{$95 \% \mathrm{CI}$} & \multirow[b]{2}{*}{ P-value } & \multirow[b]{2}{*}{ Median } & \multicolumn{2}{|c|}{$95 \% \mathrm{CI}$} & \multirow[b]{2}{*}{ P-value } \\
\hline & & Lower limit & Upper limit & & & Lower limit & Upper limit & \\
\hline Age, years & & & & 0.93 & & & & 0.35 \\
\hline$\leq 65.0$ & 5.8 & 2.7 & 8.8 & & 18.3 & 10.5 & 26.1 & \\
\hline$>65.0$ & 5.8 & 2.0 & 8.8 & & 12.5 & 6.7 & 18.2 & \\
\hline Sex & & & & 0.10 & & & & 0.64 \\
\hline Male & 5.8 & 5.1 & 6.4 & & 18.3 & 9.4 & 27.2 & \\
\hline Female & 4.6 & 0.0 & 10.6 & & 14.3 & 6.6 & 21.9 & \\
\hline Treatment & & & & 0.009 & & & & 0.00006 \\
\hline Monotherapy & 4.5 & 3.4 & 5.6 & & 7.7 & 6.3 & 9.0 & \\
\hline Combination therapy & 9.1 & 5.6 & 12.6 & & 23.0 & 17.9 & 28.9 & \\
\hline Location & & & & 0.91 & & & & 0.91 \\
\hline $\mathrm{Pbt}$ & 7.2 & 3.1 & 11.7 & & 10.5 & 0.00 & 22.1 & \\
\hline $\mathrm{Ph}$ & 5.2 & 4.1 & 6.2 & & 14.3 & 7.5 & 21.0 & \\
\hline T-stage & & & & 0.96 & & & & 0.86 \\
\hline T1-3 & 5.4 & 3.2 & 7.5 & & 19.2 & 6.0 & 32.3 & \\
\hline $\mathrm{T} 4$ & 5.8 & 2.9 & 8.6 & & 14.2 & 9.3 & 19.2 & \\
\hline $\mathrm{N}$-stage & & & & 0.87 & & & & 0.03 \\
\hline No & 5.8 & 2.8 & 8.7 & & 18.3 & 10.2 & 26.3 & \\
\hline $\mathrm{N} 1-2$ & 4.9 & 0.0 & 10.2 & & 8.2 & 5.7 & 10.6 & \\
\hline M-stage & & & & 0.01 & & & & 0.01 \\
\hline M0 & 10.4 & 5.6 & 15.2 & & 23.0 & 16.1 & 29.8 & \\
\hline M1 & 4.6 & 3.4 & 5.7 & & 10.5 & 6.5 & 13.4 & \\
\hline CEA (ng/ml) & & & & 0.16 & & & & 0.39 \\
\hline$\leq 5.0$ & 5.2 & 3.2 & 7.2 & & 12.8 & 3.7 & 21.8 & \\
\hline$>5.0$ & 5.8 & 3.0 & 8.6 & & 14.8 & 13.6 & 15.9 & \\
\hline CA19-9 (U/ml) & & & & 0.65 & & & & 0.57 \\
\hline$\leq 37.0$ & 5.8 & 0.0 & 12.8 & & & Not determir & & \\
\hline$>37.0$ & 5.8 & 3.2 & 8.4 & & 14.3 & 7.4 & 21.1 & \\
\hline Splenic volume & & & & 0.95 & & & & 0.04 \\
\hline Group 1 & 5.8 & 4.5 & 7.0 & & 14.8 & 3.6 & 25.9 & \\
\hline Group 2 & 5.8 & 0.00 & 15.2 & & 8.2 & 1.6 & 14.7 & \\
\hline
\end{tabular}

PFS, progression-free survival; OS, overall survival; CI, confidence interval; Ph, pancreatic head; Pbt, pancreatic body and tail; CEA, carcinoembryonic antigen; CA19-9, carbohydrate antigen 19-9; AST, aspartate aminotransferase; ALT, alanine aminotransferase.

system invasion) were determined using Fisher's exact test. Correlations between splenic volume and other clinical variables were evaluated using Spearman's correlation analysis. Progression-free survival (PFS) and overall survival (OS) were calculated from the date of the initial day of chemotherapy to the date of disease progression or any cause of mortality, respectively. The association of each clinicopathological parameter (age, sex, disease stage, serum levels of CEA and CA 19-9 and splenic volume) with PFS and OS was investigated. Survival analysis was performed using the Kaplan-Meier method with the log-rank test in univariate analysis. Forward stepwise multivariate analysis was performed to determine the influence of clinicopathological variables. Cox regression analysis was used for multivariate analysis, and hazard ratios (HRs) were calculated. Statistical analyses were performed using SPSS version 26.0 for Windows (SPSS Inc.), and figures were generated using Prism 7 (GraphPad, Inc.). A P-value $<0.05$ was considered to indicate a statistically significant difference.

\section{Results}

Clinical differences associated with splenic volume. The patients were divided into group $1(n=34)$ and group $2(n=16)$. The comparison of clinical characteristics between the 2 groups revealed that invasion of the portal vein system (portal vein, 
Table IV. Results of multivariate Cox regression analysis.

PFS

\begin{tabular}{|c|c|c|c|c|}
\hline \multirow[b]{3}{*}{ Variables } & \multicolumn{4}{|c|}{ PFS } \\
\hline & \multirow[b]{2}{*}{ HR } & \multicolumn{2}{|c|}{$95 \% \mathrm{CI}$} & \multirow[b]{2}{*}{ P-value } \\
\hline & & Lower limit & Upper limit & \\
\hline Chemotherapeutic regimen & & & & 0.007 \\
\hline Monotherapy & 1 & & & \\
\hline Combination therapy & 0.38 & 0.18 & 0.76 & \\
\hline M-stage, M0 (\%) & & & & 0.009 \\
\hline M1 & 1 & & & \\
\hline \multirow[t]{3}{*}{ M0 } & 0.40 & 0.20 & 0.79 & \\
\hline & \multicolumn{4}{|c|}{ OS } \\
\hline & & \multicolumn{2}{|c|}{$95 \% \mathrm{CI}$} & \\
\hline Variables & HR & Lower limit & Upper limit & P-value \\
\hline Chemotherapeutic regimen & & & & 0.0003 \\
\hline Monotherapy & 1 & & & \\
\hline Combination therapy & 0.14 & 0.05 & 0.35 & \\
\hline N-stage, N0 (\%) & & & & 0.0001 \\
\hline $\mathrm{N} 1-2$ & 1 & & & \\
\hline No & 0.35 & 0.14 & 0.91 & \\
\hline M-stage, M0 (\%) & & & & 0.0001 \\
\hline M1 & 1 & & & \\
\hline M0 & 0.17 & 0.07 & 0.42 & \\
\hline Splenic volume & & & & 0.001 \\
\hline Group 2 & 1 & & & \\
\hline Group 1 & 0.25 & 0.11 & 0.58 & \\
\hline
\end{tabular}

PFS, progression-free survival; OS, overall survival; HR, hazard ratio; CI, confidence interval.

Table V. Correlation of splenic volume and other clinical variables.

\begin{tabular}{lccccccc}
\hline & Age & Height & Weight & WBCs & NLR & CRP & Invasion score \\
\hline Spearman's r & -0.18 & -0.03 & 0.33 & -0.39 & -0.01 & 0.08 & 0.5 \\
P-value (two-tailed) & 0.21 & 0.84 & 0.02 & 0.0049 & 0.49 & 0.58 & 0.0002
\end{tabular}

WBCs, white blood cells; NLR, neutrophil-to-lymphocyte ratio; CRP, c-reactive protein. Spearman's correlation analysis was performed.

supra mesenteric vein and splenic vein) was more frequently observed in group 2 than in group 1 (group 1 vs. group 2: Portal vein invasion, 26.4 vs. $62.5 \%, \mathrm{P}=0.01$; supra mesenteric vein invasion, 32.3 vs. $75.0 \%, \mathrm{P}=0.005$; splenic vein invasion, 29.4 vs. $62.5 \%, \mathrm{P}=0.02)$. Additionally, splenic volume was larger in group 2 than in group 1 (median of $102.2 \mathrm{vs} .227 .9 \mathrm{~cm}^{3}$, $\mathrm{P}<0.001)$. No significant differences were observed in the other variables (Table II). In the survival analysis, splenic volume was related to a poor OS, while no association with PFS was observed (group 1 vs. group 2: PFS, 5.8 vs. 5.8 months, $\mathrm{P}=0.9$; OS, 14.8 vs. 8.2 months, $\mathrm{P}=0.04$ ) (Fig. 3 ).
Prognostic indicators in PC. To clarify the role of splenic volume as a prognostic indicator in $\mathrm{PC}$, uni- and multivariate analyses were conducted using several clinical variables together with splenic volume. First, univariate Kaplan-Meier survival analysis of PFS and OS was conducted. In terms of PFS, treatment (monotherapy vs. combination therapy, median of 4.5 vs. 9.1 months, $\mathrm{P}=0.009$ ) and $\mathrm{M}$-stage (M0 vs. M1, median of 10.4 vs. 4.6 months, $\mathrm{P}=0.01$ ) were found to be related to prognosis. In terms of OS, treatment (monotherapy vs. combination therapy, median of 7.7 vs. 23.0 months, $\mathrm{P}=0.00006$ ), $\mathrm{N}$-stage (N0 vs. N1-2, median of 18.3 vs. 8.2 months, $\mathrm{P}=0.03$ ), 
Table VI. Comparison of severe hematotoxicity between group 1 and group 2.

\begin{tabular}{lccc}
\hline & Group 1 (\%) & Group 2(\%) & P-value \\
\hline Leukopenia & 17.6 & 22.2 & 0.72 \\
Neutropenia & 20.6 & 18.8 & 0.99 \\
Anaemia & 0 & 0 & 1.00 \\
Thrombocytopenia & 8.8 & 37.5 & 0.02 \\
\hline
\end{tabular}

Fisher's exact test was used for these data.

Table VII. Relative dose intensity of chemotherapeutic agents.

\begin{tabular}{lccc}
\hline & Group 1 $(\%)$ & Group 2 $(\%)$ & P-value \\
\hline GEM & 64.5 & 55.8 & 0.31 \\
Nab-PTX & 59.7 & 25.2 & 0.009 \\
L-OHP & 74.0 & 55.0 & 0.39 \\
CPT-11 & 84.0 & 62.0 & 0.57 \\
5-FU & 84.0 & 93.0 & 0.78 \\
\hline
\end{tabular}

GEM, gemcitabine; Nab-PTX, nab-paclitaxel; L-OHP, oxaliplatin; CTP-11, irinotecan; 5-FU, 5-fluorouracil. Fisher's exact test was used for these data.

M-stage (M0 vs. M1, median of 23.0 vs. 10.5 months, $\mathrm{P}=0.01$ ) and splenic volume (group 1 vs. group 2, median of 14.8 vs. 8.2 months, $\mathrm{P}=0.04$ ) were related to prognosis (Table III).

The results of multivariate Cox regression analysis confirmed that treatment $(\mathrm{P}=0.007)$ and $\mathrm{M}$-stage $(\mathrm{P}=0.009)$ were significantly associated with PFS (Table IV). Additionally, treatment $(\mathrm{P}=0003), \mathrm{N}$-stage $(\mathrm{P}=0.0001), \mathrm{M}$-stage $(\mathrm{P}=0.0001)$ and splenic volume $(\mathrm{P}=0.001)$ were significantly associated with OS. Splenic volume was considered an independent prognostic factor for OS in PC (Table IV).

Clinical variables related to splenic volume. To elucidate the mechanisms through which splenic volume affects prognosis in PC, clinical variables related to splenic volume were investigated. First, the results of the correlation analysis revealed that splenic volume positively correlated to body weight $(r=0.33, P=0.02)$ and the portal system invasion score $(\mathrm{r}=0.5, \mathrm{P}=0.0002)$, but negatively correlated with the WBC count $(r=-0.39)$. No significant correlation was observed between the systemic inflammation marker, NLR, and splenic volume $(\mathrm{P}=0.49)$ (Table $\mathrm{V}$ and Fig. S1). In a comparative analysis of severe hematotoxicity, severe thrombocytopenia was more frequently observed in group 2 than in group 1 (8.8 vs. $37.5 \%, \mathrm{P}=0.02$ ) (Table VI). As consequence, treatment discontinuation was more frequently observed in group 2 compared with group 1 ( $78 \%$ vs. 47\%, P<0.0001). Finally, the RDI was compared between the 2 groups, and it was found that the RDI of nab-PTX was significantly lower in group 2 than in group 1 (median of 59.7 vs. $25.2 \%, \mathrm{P}=0.009$ ). As regards other chemotherapeutic agents, no significant differences were observed between the 2 groups (Table VII).

\section{Discussion}

In the present study, it was demonstrated that a large splenic volume was an independent prognostic indicator of the OS of patients with PC, which may be attributed to an increased incidence of severe thrombocytopenia and a reduced RDI of nab-PTX. To the best of our knowledge, the present study is the first to clarify the prognostic role of splenic volume in patients with PC treated with chemotherapy and elucidate the potential underlying mechanism.

$\mathrm{PC}$ is one of the most lethal malignancies worldwide, as the majority of PC cases are not indicated for curative resection at the time of diagnosis and are treated with palliative chemotherapy $(11,12)$. At the present time, several regimens are considered to be standard treatments, and practitioners can select one of these as an initial treatment considering the patient's general condition (13-15). With combination therapy, such as FFX or GnP, the median OS period can be expected to be almost 1 year even in patients with metastatic disease; however, the management of severe hematotoxicity, febrile neutropenia or spontaneous bleeding caused by severe thrombocytopenia is important for physicians to obtain the maximum benefit of chemotherapy. Currently, UGT1A1 polymorphism is the only marker utilized in routine practice to predict adverse events in patients who are treated with a CPT-11-containing regimen such as FOLFIRINOX $(16,17)$. On the other hand, UGT1A1 polymorphism is not useful in other regimens, and no predictive factor exists at this time.

Splenic volume in patients with solid tumors may be influenced by sex, age and underlying chronic inflammation $(10,18)$. Chronic inflammation has been considered to be linked to tumor progression in various types of cancer, since it can promote cancer growth and negatively affect the immune system by inducing the activation of immune suppressor cells (19-22). However, the present study could not find an association between splenic volume and systemic inflammation indices (e.g., WBC, CRP and NLR). On the other hand, splenic volume positively correlated with the invasion score, which indicated that an enlarged splenic volume may be derived from left-sided portal hypertension caused by PC vascular invasion. As a consequence of splenic sequestration, severe thrombocytopenia was considered to be frequently observed in patients with an enlarged splenic volume, which may influence the decreased RDI of chemotherapeutic agents. Considering the decreased RDI for the 1st line treatment, it was hypothesized that PFS may be affected by splenic volume. However, splenic volume was shown to be an independent prognostic indicator for OS, but not for PFS. Since there were no significant differences in most of the clinical variables between the 2 groups, the mechanisms through which splenic volume influences OS are not clear. Perhaps non-hematologic adverse events were more frequently observed in patients with larger splenic volumes during 1st-line treatment, and the patients eventually could not tolerate further treatment after treatment failure.

The present study had several limitations. First, the present study was conducted at a single referral center, and the results may not be generalizable to all patients with PC. The small sample size also limited the reliability of our statistical analysis. Second, the present study could not evaluate the prognostic role of splenic volume for each chemotherapeutic regimen since the number of patients was limited. Therefore, additional studies 
including a larger number of patients with various clinical backgrounds are warranted.

In conclusion, splenic volume may be a predictive factor for severe thrombocytopenia and may be a long-term prognostic indicator for patients with PC.

\section{Acknowledgements}

The authors gratefully acknowledge the work of past and present members of the Department of Gastroenterology, Fukushima Medical University School of Medicine, Fukushima, Japan.

\section{Funding}

No funding was received.

\section{Availability of data and material}

All data generated or analyzed during the present study are included in the published article.

\section{Authors' contributions}

RS designed the study. RS and SI wrote the manuscript. RS, SI and HW analyzed the data. RS, SI, HW, TT, MS, YS, JN, MT, TK, MH, TH, HI and HO contributed to patient care. All authors read and approved the final manuscript.

\section{Ethics approval and consent to participate}

The study protocol conformed to the ethical guidelines of the 1975 Declaration of Helsinki and was approved by the institutional Review Committee of Fukushima Medical University (Fukushima, Japan; IRB \#29254). The institutional review board waived the need for written informed patient consent because of the retrospective and non-interventional nature of the study.

\section{Patient consent for publication}

Not applicable.

\section{Competing of interests}

The authors declare that they have no competing interests.

\section{References}

1. Hwang TL, Jan YY, Jeng LB, Chen MF, Hung CF and Chiu CT: The different manifestation and outcome between pancreatitis and pancreatic malignancy with left-sided portal hypertension. Int Surg 84: 209-212, 1999.

2. Koklu S, Yuksel O, Arhan M, Coban S, Başar O, Yolcu OF, Uçar E, Ibiş M, Ertugrul I and Sahin B: Report of 24 left-sided portal hypertension cases: A single-center prospective cohort study. Dig Dis Sci 50: 976-982, 2005.

3. Sakorafas GH, Sarr MG, Farley DR and Farnell MB: The significance of sinistral portal hypertension complicating chronic pancreatitis. Am J Surg 179: 129-133, 2000.

4. Turrill FL and Mikkelsen WP: 'Sinistral' (left-sided) extrahepatic portal hypertension. Arch Surg 99: 365-368, 1969.

5. Hubert C, Sempoux C, Humblet Y, van den Eynde M, Zech F, Leclercq I and Gigot JF: Sinusoidal obstruction syndrome (SOS) related to chemotherapy for colorectal liver metastases: Factors predictive of severe SOS lesions and protective effect of bevacizumab. HPB (Oxford) 15: 858-864, 2013.
6. Overman MJ, Maru DM, Charnsangavej C, Loyer EM, Wang H, Pathak P, Eng C, Hoff PM, Vauthey JN, Wolff RA and Kopetz S: Oxaliplatin-mediated increase in spleen size as a biomarker for the development of hepatic sinusoidal injury. J Clin Oncol 28: 2549-2555, 2010.

7. Aarnink A, Richard C, Truntzer C, Vincent J, Bengrine L, Vienot A, Borg C and Ghiringhelli F: Baseline splenic volume as a surrogate marker of FOLFIRINOX efficacy in advanced pancreatic carcinoma. Oncotarget 9: 25617-25629, 2018.

8. Common Terminology Criteria for Adverse Events(CTCAE), version 5.0. US Department of Health and Human Services, 2017. https://ctep.cancer.gov/protocoldevelopment/electronic_applications/docs/CTCAE_v5_Quick_Reference_5x7.pdf. Accessed November 27, 2017.

9. Kamarajah SK, Burns WR, Frankel TL, Cho CS and Nathan H: Validation of the American joint commission on cancer (AJCC) 8th edition staging system for patients with pancreatic adenocarcinoma: A surveillance, epidemiology and end results (SEER) analysis. Ann Surg Oncol 24: 2023-2030, 2017.

10. Harris A, Kamishima T, Hao HY, Kato F, Omatsu T, Onodera Y, Terae S and Shirato H: Splenic volume measurements on computed tomography utilizing automatically contouring software and its relationship with age, gender, and anthropometric parameters. Eur J Radiol 75: e97-e101, 2010.

11. Global Burden of Disease Cancer Collaboration; Fitzmaurice $C$, Abate D, Abbasi N, Abbastabar H, Abd Allah F, Abdel Rahman O, Abdelalim A, Abdoli A, Abdollahpour I, et al: Global, regional, and national cancer incidence, mortality, years of life lost, years lived with disability, and disability-adjusted life-years for 29 cancer groups, 1990 to 2017: A systematic analysis for the global burden of disease study. JAMA Oncol 5: 1749-1768, 2019.

12. Siegel RL, Miller KD and Jemal A: Cancer statistics, 2019. CA Cancer J Clin 69: 7-34, 2019.

13. Conroy T, Desseigne F, Ychou M, Bouché O, Guimbaud R, Bécouarn Y, Adenis A, Raoul JL, Gourgou Bourgade S, de la Fouchardière $\mathrm{C}$, et al: FOLFIRINOX versus gemcitabine for metastatic pancreatic cancer. N Engl J Med 364: 1817-1825, 2011.

14. Von Hoff DD, Ervin T, Arena FP, Chiorean EG, Infante J, Moore M, Seay T, Tjulandin SA, Ma WW, Saleh MN, et al: Increased survival in pancreatic cancer with nab-paclitaxel plus gemcitabine. N Engl J Med 369: 1691-1703, 2013.

15. Ueno H, Ioka T, Ikeda M, Ohkawa S, Yanagimoto H, Boku N, Fukutomi A, Sugimori K, Baba H, Yamao K, et al: Randomized phase III study of gemcitabine plus S-1, S-1 alone, or gemcitabine alone in patients with locally advanced and metastatic pancreatic cancer in Japan and Taiwan: GEST study. J Clin Oncol 31: 1640-1648, 2013.

16. Shirasu H, Todaka A, Omae K, Fujii H, Mizuno N, Ozaka M, Ueno H, Kobayashi S, Uesugi K, Kobayashi N, et al: Impact of UGT1A1 genetic polymorphism on toxicity in unresectable pancreatic cancer patients undergoing FOLFIRINOX. Cancer Sci 110: 707-716, 2019.

17. Takahara N, Nakai Y, Isayama H, Sasaki T, Satoh Y, Takai D, Hamada T, Uchino R, Mizuno S, Miyabayashi K, et al: Uridine diphosphate glucuronosyl transferase 1 family polypeptide A1 gene (UGT1A1) polymorphisms are associated with toxicity and efficacy in irinotecan monotherapy for refractory pancreatic cancer. Cancer Chemother Pharmacol 71: 85-92, 2013.

18. Prassopoulos P, Daskalogiannaki M, Raissaki M, Hatjidakis A and Gourtsoyiannis N: Determination of normal splenic volume on computed tomography in relation to age, gender and body habitus. Eur Radiol 7: 246-248, 1997.

19. Gabrilovich DI: Myeloid-derived suppressor cells. Cancer Immunol Res 5: 3-8, 2017.

20. Kumar V, Patel S, Tcyganov E and Gabrilovich DI: The nature of myeloid-derived suppressor cells in the tumor microenvironment. Trends Immunol 37: 208-220, 2016.

21. Padoan A, Plebani M and Basso D: Inflammation and pancreatic cancer: Focus on metabolism, cytokines, and immunity. Int J Mol Sci 20: 676, 2019.

22. Shalapour S and Karin M: Pas de Deux: Control of anti-tumor immunity by cancer-associated inflammation. Immunity 51 : 15-26, 2019.

This work is licensed under a Creative Commons Attribution-NonCommercial-NoDerivatives 4.0 International (CC BY-NC-ND 4.0) License. 